\title{
SENSIBILITY and RESISTANCE of genus Haemophilus to ophthalmic antibiotics in newborns:
} 152 cases

\author{
Franco Giardini', Laura Tonini², Cristina Pollino', Giuseppe Grandi', Tullio Albanesi', \\ Luisa Indovina ${ }^{3}$, Angela Vaira Maria ${ }^{3}$ \\ I Laboratorio Analisi, Dipartimento dei Servizi, Ospedale Oftalmico, Torino \\ 2 Oftalmologia Infantile, Dipartimento di Oftalmologia, Ospedale Oftalmico, Torino \\ 3 Servizio di Ortottica, Dipartimento di Oftalmologia, Ospedale Oftalmico, Torino
}

Key words: Oftalmologia pediatrica, Haemophilus, Dacriostenosi

Sensibilità e resistenze del genere Haemophilus agli antibiotici di uso topico oftalmico in pazienti della prima infanzia: I52 casi

\section{INTRODUZIONE}

Le infezioni oculari nell'età compresa tra 0 e 2 anni sono abbastanza frequenti poichè nella primissima infanzia le difese quali lisozima, lattoferrina, immunoglobuline secretorie e defensine non si sono ancora completamente stabilizzate.

Le infezioni oculari che interessano la prima infanzia sono caratterizzate principalmente in ordine di frequenza dalle congiuntiviti, dalle patologie del sacco lacrimale e dalle cheratiti.

Le congiuntiviti della prima infanzia possono essere acute o croniche, solitamente catarrali, possono avere come agenti etiologici patogeni diversi tra i quali spiccano Haemophilus, Streptococcus pneumoniae e gli Adenovirus in percentuale che talora sfiorano il $30 \%$ per ciascuno. Le patologie del sacco lacrimale o dacriostenosi congenite, per mancato o ritardato sviluppo del canale nasolacrimale, sono frequenti dal $3^{\circ}$ mese all'anno di età, molto più sporadiche successivamente, solitamente monolaterali, e tendono a risolversi spontaneamente entro l'anno. Se ciò non avviene si procede a lavaggi delle vie lacrimali in lieve narcosi. Esse causano frequenti congiuntiviti per l'epifora persistente accompagnata dal reflusso muco-purulento dal puntino lacrimale inferiore.

Gli agenti patogeni più frequentemente interessati sono anche qui Haemophilus e Streptococcus pneumoniae, nonchè Pseudomonas e Stafilococchi Coagulasi Negativi.

\section{METODI}

Per questa indagine sono stati considerati $\mathrm{i}$ pazienti infanti afferiti al nostro Pronto Soccorso, al Servizio di Ortottica o direttamente al nostro Laboratorio Analisi dal 1/01/2011 al 30/06/2011.
In totale sono stati arruolati (Tabella 3) 902 pazienti infanti positivi all'esame colturale, tra $i$ quali vi erano 152 isolamenti di schizomiceti del gen. Haemophilus (130 ceppi di H. influenzae e 22 di $H$. parainfluenzae) (Tabella 1 ).

Ogni tampone, dopo esser stato passato nei fornici congiuntivali inferiori, veniva seminato su Agar Cioccolato, su Agar Sangue Columbia e in un brodo Cuore Cervello (aerobiosi a $37^{\circ} \mathrm{C}$ ), nonchè su Agar Haemophilus (microaerofilia a $37^{\circ} \mathrm{C}$ ).

\section{RISULTATI}

I batteri del gen. Haemophilus isolati (pari al $16.8 \%$ degli isolamenti colturali totali) hanno dato le seguenti percentuali di sensibilità in vitro con il metodo Kirby-Bauer, riferite agli antibiotici per uso topico oculare saggiati: Ampicillina $(50.00 \%)$, Cloramfenicolo (96.1\%), Netilmicina $(65.8 \%)$, Ofloxacina $(94.1 \%)$, Ac.Fusidico $(3.3 \%)$, Tobramicina $(18.1 \%)$, Norfloxacina (93.8\%), Lomefloxacina (86.00\%), Levofloxacina (87.8\%), Neomicina (20.9\%), Ciprofloxacina $(92.1 \%)$, Amikacina (36.1\%), Gentamicina $(36.2 \%)$, Tetraciclina $(80.3 \%)$, Vancomicina (3.2\%), Moxifloxacina (3.7\%) (Tabella 2).

\section{CONCLUSIONI}

I risultati ottenuti evidenziano numerose resistenze ai comuni antibiotici prescritti sotto forma di colliri o pomate per la cura delle infezioni oculari infantili. Si evince quindi la necessità di eseguire l'esame colturale ogniqualvolta si presentino pazienti infanti affetti da infezioni oculari di gravità diverse al fine di individuarne l'agente patogeno e poter effettuare un antibiogramma mirato per un corretto trattamento antibatterico. 
Tabella I. Isolamenti di Haemophilus per patologia.

\begin{tabular}{lcccc}
\hline CEPPI BATTERICI & CONGIUNTIVITI & PATOLOGIE DACRIO & ULCERE & TOTALE \\
\hline Haemophilus influenzae & 76 & 67 & $I$ & 144 \\
\hline Haemophilus parainfluenzae & 6 & 2 & 1 & 8 \\
\hline & & & & 152 \\
\hline
\end{tabular}

Tabella 2. Sensibilità verso I I52 ceppi del gen. Haemophilus ai seguenti antibiotici per uso topico oculare.

\begin{tabular}{lc}
\hline & SENSIBILITA் \\
\hline AMPICILLINA & $50 \%$ \\
\hline CLORAMFENICOLO & $96.1 \%$ \\
\hline NETILMICINA & 65.8 \\
\hline OFLOXACINA & 94.1 \\
\hline AC.FUSIDICO & $3.3 \%$ \\
\hline TOBRAMICINA & $18.1 \%$ \\
\hline NORFLOXACINA & $93.8 \%$ \\
\hline LOMEFLOXACINA & $86 \%$ \\
\hline LEVOFLOXACINA & $\mathbf{8 7 . 8 \%}$ \\
\hline NEOMICINA & $20.9 \%$ \\
\hline CIPROFLOXACINA & $92.1 \%$ \\
\hline AMIKACINA & $36.1 \%$ \\
\hline GENTAMICINA & 36.2 \\
\hline TETRACICLINA & $\mathbf{8 0 . 3} \%$ \\
\hline VANCOMICINA & $3.2 \%$ \\
\hline MOXIFLOXACINA & $\mathbf{9 3 . 7 \%}$ \\
\hline
\end{tabular}

\section{BIBLIOGRAFIA}

1. Bourcier T, et al. Bacterial keratitis: predisposing factors, clinical and microbiological rewiew of 300 cases. Br J Ophthalmol 2003; 87: 834-8.

2. Grandi G, Grignolo FM, Giardini F. Manuale di
Tabella 3.

Elenco totale dei ceppi batterici isolati (902)

\begin{tabular}{lc}
\hline MICROORGANISMO & N. CASI \\
\hline Streptococcus pneumoniae & 200 \\
\hline Streptococco alfa-emolitico & 30 I \\
\hline Gen. Haemophilus & I52 \\
\hline Gen. Moraxella & 80 \\
\hline S. aureus & 72 \\
\hline Gen. Pseudomonas & 27 \\
\hline Cons & 32 \\
\hline Gen. Klebsiella & 7 \\
\hline Streptococco beta-emolitico & 9 \\
\hline Gen. Pasteurella & I I \\
\hline Gen. Serratia & 4 \\
\hline MRSA & I \\
\hline E.coli & 2 \\
\hline Citrobacter diversus & 2 \\
\hline Proteus vulgaris & I \\
\hline Candida krusei & I
\end{tabular}

Microbiologia Oculare. Ed Medical Systems Spa Genova, Settembre 2011.

3. Wong VW, et al. Pediatric ocular surface infections: a 5 years rewiew of demographics, clinical features, risk factors, microbiological results and treatment. Cornea. 2011 sep; 30 (9): 995-1002. 